\title{
Chemically Specific Multiscale Modeling of the Shear-Induced Exfoliation of Clay-Polymer Nanocomposites
}

\author{
James L. Suter ${ }^{*} \dagger$ and Peter V. Coveney* ${ }^{*}+0$ \\ ${ }^{\dagger}$ Centre for Computational Science and ${ }^{\ddagger}$ Centre for Computational Science, University College London, 20 Gordon Street, London \\ WC1H 0AJ, United Kingdom
}

\section{Supporting Information}

\begin{abstract}
We recently showed, using chemically specific modeling and simulation, how the process of intercalation of polymers within clay sheets occurs, transforming the largescale materials properties by a specific set of spatial and temporal processes that can lead to exfoliation. Here, we use the same hierarchal multiscale modeling scheme to understand the processes that occur during the shear-induced processing of clay-polymer nanocomposites. For both hydrophobic polymers (polyethylene) and hydrophilic polymers (poly(ethylene glycol)), we used free-energy methods to identify the lowest-free-energy separation of the clay sheets; the polymer molecules spontaneously intercalate into the clay interlayer from the surrounding polymer melt. We apply shear forces to investigate exfoliation and find that while exfoliation is promoted by shearing, it is the surfactant molecules that play the dominant role in resisting it.
\end{abstract}

\section{INTRODUCTION}

The central goal of theory, modeling, and simulation in materials science and engineering is to predict the large-scale properties of materials on the basis of chemical composition and processing conditions. ${ }^{1-3}$ In the field of nanocomposites, we would like to be able to predict whether it is possible to disperse the solid phase; for clay-polymer nanocomposites, and other two-dimensional nanomaterials, improvement in the elastic properties of the nanocomposite is greatest when the two-dimensional layers are fully dispersed, exposing the maximum surface area to the surrounding polymeric matrix. ${ }^{4-7}$ The difficulty lies in the small spacing between the clay layers, which is of order $1 \mathrm{~nm}$ (compared to lateral dimensions of 10 $\mathrm{nm}$ to microns). Grafting surfactants to clay surfaces is a common step in the processing of clay-polymer nanocomposites. This approach facilitates the delamination and exfoliation of clay minerals when they are aggregated into stacks, called tactoids ${ }^{4,5}$ by increasing the gallery spacing and allowing polymer penetration. Shear forces are also often required, however, to remove individual clay layers from the tactoid through methods such as extrusion processing.

There is, therefore, much interest in determining the conditions that increase the gallery spacing and promote exfoliation. Previous theoretical efforts in examining the thermodynamics of clay-polymer nanocomposites include lattice mean-field models developed by Vaia and Giannelis, ${ }^{8,9}$ which calculated the enthalpic and entropic contributions to the expansion of surfactant-treated two-clay-layer systems and their subsequent penetration by long-chain polymers. They showed that there is a substantial loss of entropy for confined intercalating polymers, which can be compensated by an increase in enthalpy for properly selected surfactants. Subsequently, Balazs and co-workers applied lattice selfconsistent field theory (SCFT) to examine nanocomposite morphologies as a function of the density and length of the surfactants and polymer molecular weight. ${ }^{10-12}$ They found that even for negative Flory-Huggins interaction parameters between the components, for long polymer chains, only intercalated nanocomposites were formed. For low surfactant molecular weight and for the surfactant and polymer possessing the same chemistry, the system was found to be immiscible.

However, such models lack atomistic detail, being defined by polymer-mediated contributions to the particle-pair potential. There could be contributions from longer-range clay-clay interactions and excluded volume interactions, as shown by molecular dynamics simulations of clay surfaces. ${ }^{13-17}$ In this study, we build upon our previous work studying the intercalation of polymer molecules into clay tactoids ${ }^{2}$ to perform detailed molecular modeling of clay-polymer nanocomposites to address the exfoliation of these systems. We use large-scale coarse-grained molecular dynamics (CG-MD) to observe, for the first time, the process of intercalation and the formation of intercalated and exfoliated polymer-clay composite systems. Here, we apply these methods to examine the role of surfactants and polymers in the exfoliation process. Surfactants not only reduce the adhesion between layers but

Received: March 21, 2018

Accepted: June 4, 2018

Published: June 15, 2018 
(a)

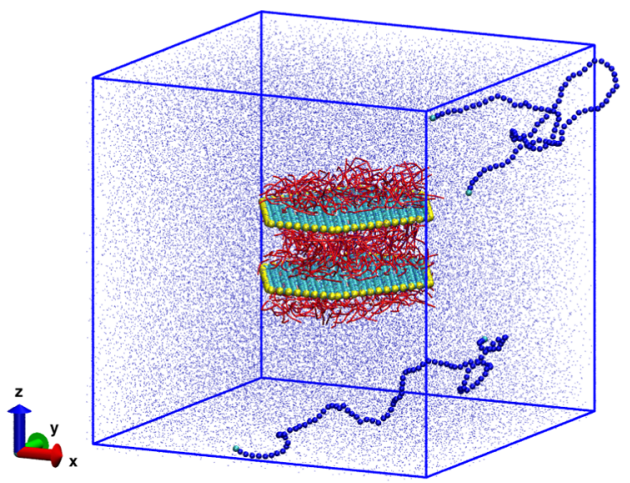

(b)

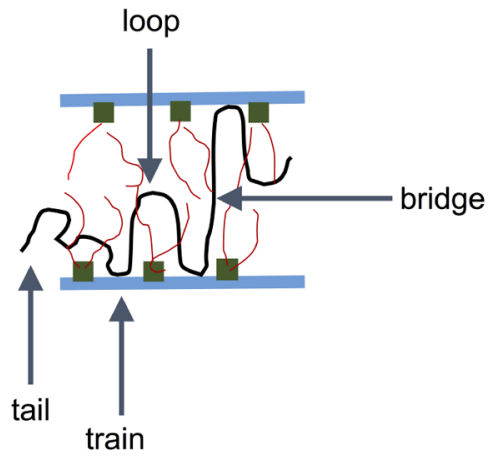

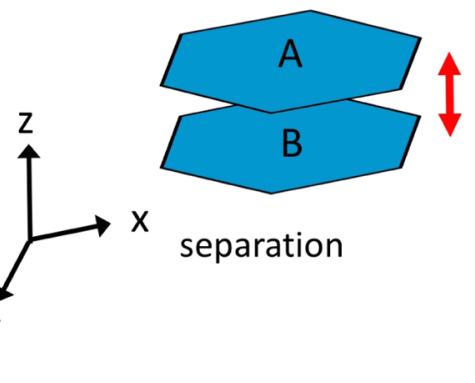

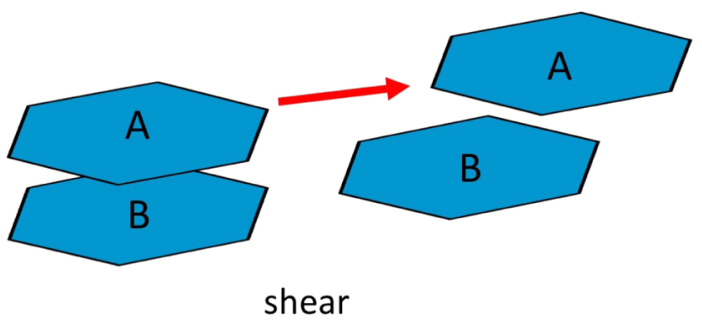

(c)

Figure 1. (a) Initial setup of the clay-polymer CG system used in this study. The surfactant molecules are shown in red, clay CG pseudoatoms are cyan, and clay edge CG pseudoatoms are yellow. The two-clay-layer system is in the center of a box of molten polymer (each polymer CG atom is represented by a blue dot). Two highlighted polymer molecules illustrate the amorphous nature of the polymer matrix (blue spheres). The dimensions of the simulation box are approximately $200 \times 200 \times 200 \AA^{3}$. (b) Diagram illustrating the different configurations of adsorbed polymers and surfactants in the interlayer spacing. The clay layers are shown as thick blue lines, the surfactant head groups as brown squares, the surfactant tails as red lines, and the adsorbed polymers as black lines. Surfactant tails can interact with surfactant tails that are attached, via their head groups, to the opposite surface, to effectively form bridges across the interlayer spacing. (c) A schematic diagram illustrating our procedure for computing the free energy of separation (reaction coordinate is the red line) and shear simulations of clay layers A and B (force is applied to clay layer A in the $x$ direction). Only the clay layers are illustrated; surfactant and polymer molecules are not shown.

also alter their lubrication and friction properties. ${ }^{18-21}$ The shear stress required to delaminate the clay layers must overcome the residual adhesive forces to form exfoliated particles. $^{22-24}$ In the case of intercalated clay layers, these adhesive forces result from a combination of surfactant and polymer molecules, which reside on the surface of the clay as well as within the interlayer spacing. The role of the adsorbed and free polymer in determining the exfoliation properties of clay layers has been hitherto unknown.

We use the coarse-grained $\mathrm{MD}$ technique to simulate a clay platelet immersed in a reservoir of polymer melt (see Figure 1a) to address this problem. No assumptions about the equilibrium separation of the clay platelets and the density of polymer molecules within the interlayer are made. The chemical potential of the surrounding polymer melt is therefore included in our simulation, and it is this that drives the evolution of the system, including intercalation and shearinduced exfoliation. As the polymer molecules intercalate, they adopt conformations, which are partially within the clay interlayer spacing and partially outside (see Figure $1 \mathrm{~b}$ ).

We use free-energy methods to calculate the equilibrium separation between clay sheets and then investigate the shear force required to exfoliate a clay layer. In this way, we are able to dissect those processes, which promote and inhibit exfoliation.

\section{MODELING METHODS}

To exemplify shear-induced polymer-driven exfoliation of organophilic clays in a chemically specific manner, we study montmorillonite clay with a variety of quaternary ammonium surfactants and molten polymers. We consider two polymers for the matrix: hydrophilic poly(ethylene glycol) (PEG) ${ }^{25-28}$ and hydrophobic polyethene (PE). For surfactants, we consider the dimethyldioctadecylammonium ionic surfactant, denoted $\mathrm{NC18}$, which has two long hydrophobic alkyl chains (each of length $\mathrm{C}_{18}$, chemically identical to the $\mathrm{PE}$ polymer) and a surfactant containing two chains made of PEG monomer units (hence, chemically identical to the free PEG polymer), denoted $\mathrm{NPEG}_{2}$. The quaternary ammonium ions interact strongly with the charged sites on the clay surface and can be considered to be tethered surfactants. All polymer molecules used in this study are 100 monomer units long, ensuring that we are in the entanglement regime for the polymer molecules. All simulations were performed at the elevated temperature of $500 \mathrm{~K}$ and a pressure of $100 \mathrm{~atm}$, associated with melt processing.

The procedure to create the CG interaction parameters has been described in our previous studies. ${ }^{1,2}$ The CG interaction parameters are calculated from numerous small atomistic simulations and are designed to match structural details including radial distributions functions and density profiles perpendicular to the clay surface ${ }^{1}$ using Iterative Boltzmann Inversion. ${ }^{29-31}$ Each clay layer consists of $672 \mathrm{CG}$ pseudoatoms with lateral dimensions of approximately $140 \times$ 
$100 \AA^{2}$. To examine the role of clay surface charge density, which in turn affects the density of charge-balancing surfactants on the clay surface, we consider four different isomorphic substitution percentages in the clay framework. These correspond to substituting $\mathrm{Al}^{3+}$ ions with $\mathrm{Mg}^{2+}$ ions in the octahedral sheet of the clay framework. Table 1 lists various aspects of the models used in the study and the nomenclature we use to identify them.

Table 1. Organoclay Models Studied in this Study, Where Substitution Rate Refers to a Ratio of $\mathrm{Mg}^{2+}$ to Octahedral Ions in the Clay Framework and Consequently the Number of Surfactant Molecules Tethered to the Clay Surface ${ }^{a}$

\begin{tabular}{clcc}
$\begin{array}{c}\text { substitution rate } \\
(\%)\end{array}$ & $\begin{array}{c}\text { surfactant } \\
\text { type }\end{array}$ & $\begin{array}{c}\text { polymer } \\
\text { type }\end{array}$ & $\begin{array}{c}\text { lowest-free-energy } \\
\text { separation }(\AA)\end{array}$ \\
9 & $\mathrm{Na}^{+}$ & PEG & 19 \\
9 & $\mathrm{NC}_{2} 8_{2}$ & PEG & 42 \\
12.5 & $\mathrm{NC}_{2} 8_{2}$ & PEG & 44 \\
17.5 & $\mathrm{NC18}_{2}$ & PEG & 45 \\
9 & $\mathrm{NC}_{2}$ & PEG & 39 \\
9 & $\mathrm{NPEG}_{2}$ & $\mathrm{PEG}$ & 24 \\
9 & $\mathrm{NC}_{2}$ & $\mathrm{PE}$ & 26,28 \\
\hline
\end{tabular}

${ }^{a}$ The lowest-free-energy separation is calculated using umbrella sampling, with the reaction coordinate perpendicular to the clay sheet.

A snapshot of the two-clay-layer system used is shown in Figure 1a. Each simulation contains approximately 77000 CG particles, corresponding to 540000 atoms. The simulation box is approximately $200 \AA^{3}$, and the polymers are built using a Monte-Carlo growth program, we described previously. ${ }^{1}$ There are 717 polymer molecules in each model. The density of the polymer phase is approximately $0.62 \mathrm{~g} / \mathrm{mL}$, assuming $20 \%$ of the simulation box volume is occupied by the clay platelet. All simulations were performed using the LAMMPS code. ${ }^{32}$ To calculate the Gibbs free energy, the difference in the $z$ coordinates of clay layers A and B, i.e., the separation between the basal planes of the clay layers (see Figure 1c), is used as the reaction coordinate. Umbrella sampling is used to calculate the free energy as a function of layer separation, with the free energy reconstructed using the WHAM method. ${ }^{33}$ For the shear simulations, a snapshot from the basal-plane separation simulation is selected at the lowest-free-energy separation. One clay layer is fixed, and a force is imposed equally to all atoms in the other clay layer in the $x$ direction at every timestep of the molecular dynamics simulation. We observe how far the clay layer A moves with this additional force by calculating the difference in the $x$ coordinates of the center of mass of the clay layers. For more details on our simulation procedure, see Supporting Information (SI).

\section{RESULTS AND DISCUSSION}

In the following section, we examine the changes that occur in the interlayer gallery during expansion and describe the consequences of the configurations of adsorbed polymer and surfactant molecules for resisting the shear-induced separation of clay layer.

Free Energy of Basal-Plane Separation. Our simulations show the following results: when the surrounding polymer is hydrophilic (PEG), and there are no surfactant molecules in the clay galleries (only $\mathrm{Na}^{+}$ions), the thermodynamically lowestenergy state is for a bilayer of polymer to intercalate. When the surfactant and the polymer are chemically identical (either PEG or PE based), the clay galleries expand to form a tri- or quadlayer in the clay interlayer spacing, but there is no polymer density in the center of the clay galleries. The surfactant and the polymer molecules are immiscible, and intercalation is restricted to the very edges of the clay tactoid. This immiscibility is consistent with the SCFT model of Balazs et al., ${ }^{10}$ although we observe a slight increase from the unintercalated $d$-spacing $(\sim 23 \AA)$ due to small amount of polymer intercalation on the edges of the clay. When the surrounding polymer is hydrophilic and the surfactant tails are hydrophobic (i.e., PE based), the clay galleries expand considerably, the surfactant molecules lengthen to almost their full extent, and the surrounding polymer intercalates onto the surface of the clay sheet, forming up to six layers in the interlayer spacing (see SI for atomic density profiles perpendicular to the clay surface). These results are shown in Figure 2. It should be noted that there are valleys and peaks in the high-energy regions in the free-energy profiles; these are poorly sampled regions, so we cannot draw any conclusions
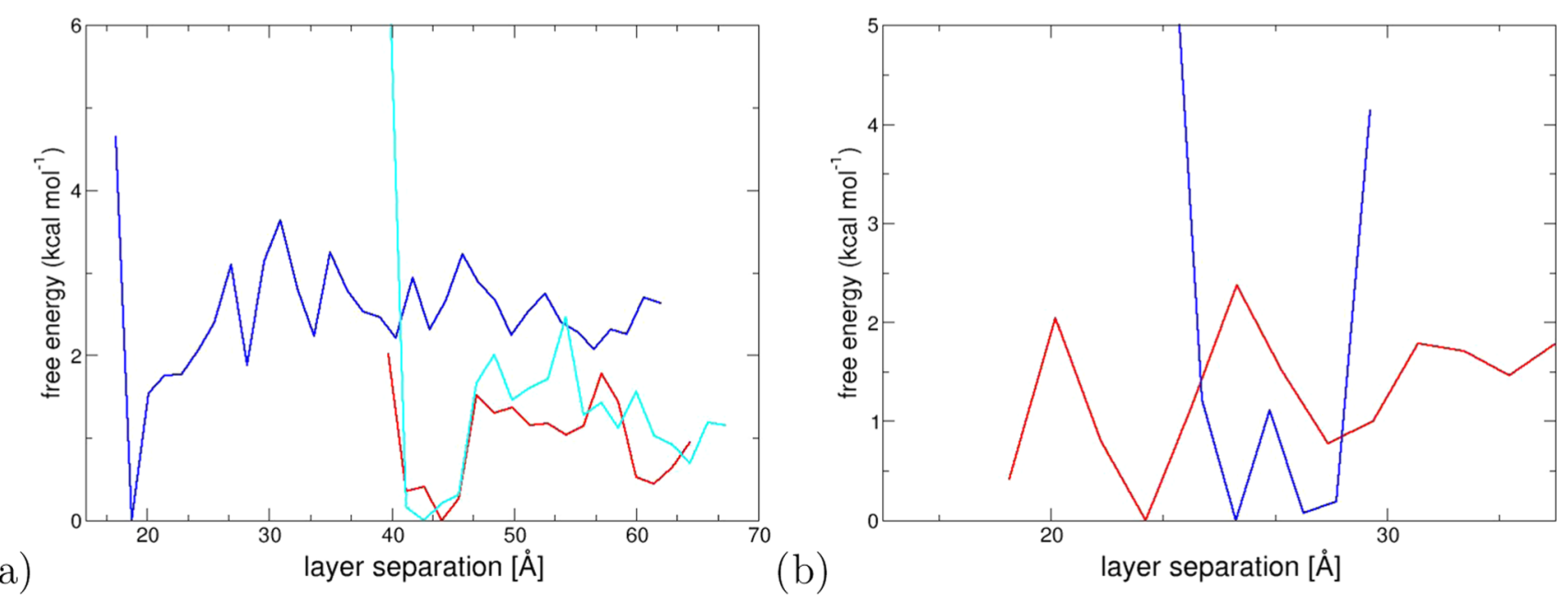

Figure 2. Free energies of clay-layer separation. (a) Hydrophilic polymer systems: blue line, $\mathrm{Na}^{+}$and PEG polymer; red line, $\mathrm{NC}_{2} 8_{2}$ surfactant and PEG polymer ( $9 \%$ substitution rate); light blue line, $\mathrm{NC}_{1} 8_{2}$ surfactant and $\mathrm{PEG}$ polymer ( $12.5 \%$ substitution rate). (b) Identical surfactant backbone and polymer systems: red line, $\mathrm{NPEG}_{2}$ surfactant and $\mathrm{PEG}$ polymer; blue line, $\mathrm{NC}_{2} 8_{2}$ surfactant and $\mathrm{PE}$ polymer. 


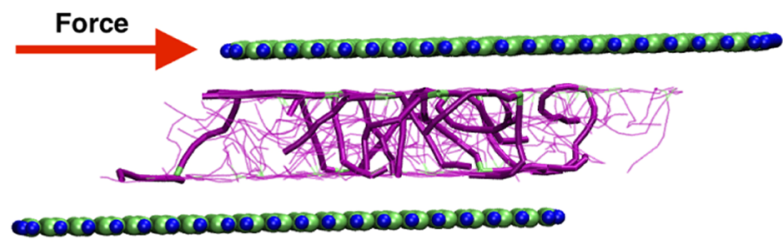

a

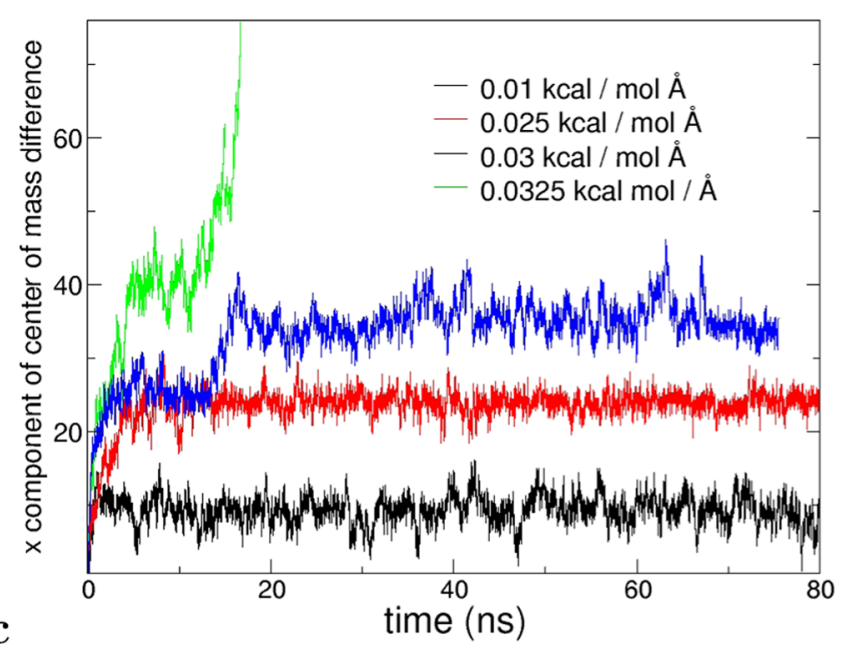

b
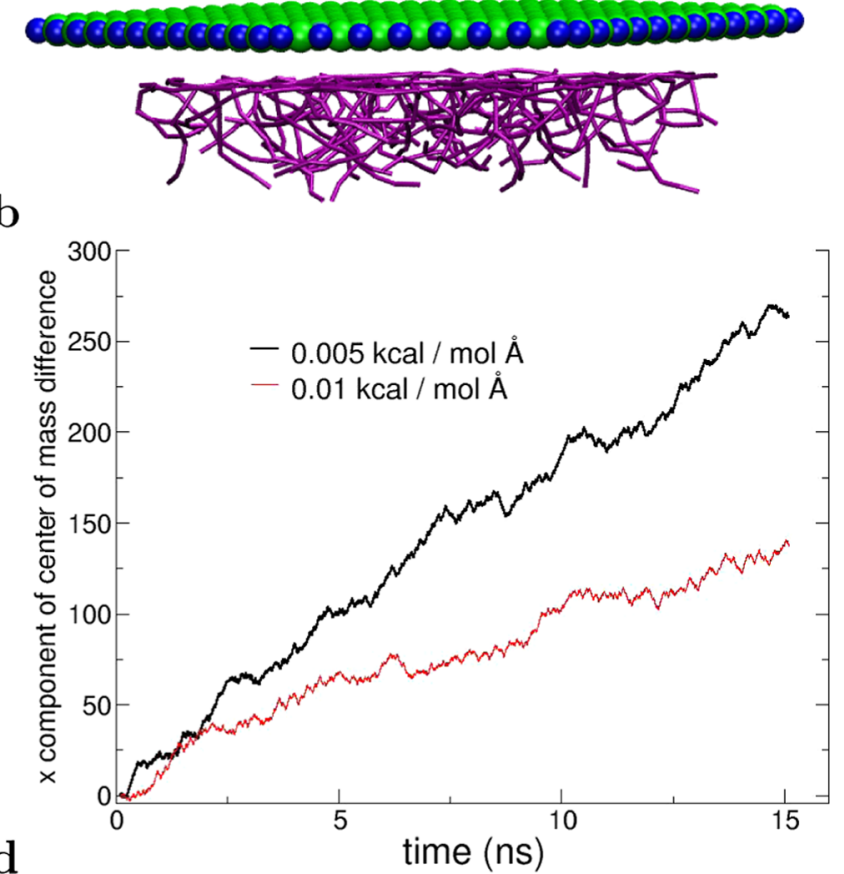

Figure 3. Shear-induced exfoliation of the $\mathrm{NC}_{1} 8_{2}$ surfactant and PE polymer system. (a) Illustration of our shear-induced exfoliation simulation for the PE polymer and $\mathrm{NC}_{1} 8_{2}$ surfactant system at a $d$-spacing of $28 \AA$. The highlighted surfactant molecules bridge between the clay layers. (b) A snapshot from simulation of one of the clay layers with noninteracting clay layers ( $d$-spacing separation of $48 \AA$ ). The difference in $x$ component of the center of mass of clay layers during the shearing simulations as a function of force added to each atom in the upper clay layer applied at each timestep for a $d$-spacing of (c) $28 \AA$ and (d) $48 \AA$. The colors in (c) are as follows: black, $0.01 \mathrm{kcal} /(\mathrm{mol} \AA)$; red, $0.025 \mathrm{kcal} /(\mathrm{mol} \AA)$; blue, 0.03 $\mathrm{kcal} /(\mathrm{mol} \AA)$; green, $0.0325 \mathrm{kcal} /(\mathrm{mol} \AA)$. The colors in $(\mathrm{d})$ are as follows: black, $0.005 \mathrm{kcal} /(\mathrm{mol} \AA)$; red, $0.01 \mathrm{kcal} /(\mathrm{mol} \AA)$.

about the shape of profile at these separations. With a longer length of simulation between each $d$-spacing point in the umbrella sampling, it might be possible to have more confidence in these high-energy areas, but the time scale of our simulations is sufficient to draw conclusions on the position and shape of the lowest-free-energy regions in the free-energy profile, as discussed above.

The behavior described above can be understood by considering the balance between the various thermodynamic contributions. ${ }^{8,9}$ When the enthalpic interactions between the clay surface and the surfactants are the same as those of the polymer and the clay surface, there is an entropic penalty for the polymer molecules to intercalate into the confined space between the clay sheets, and hence, gallery expansion is unfavorable. When the interaction between the polymer and the clay sheet is favorable, this entropic penalty is overcome, and the clay sheet interlayer spacing increases to accommodate the polymer molecules. There is also a gain in conformational entropy of the surfactants, which can now extend further from the surface. However, at a distance of 40-50 $\AA$, the surfactant molecules are almost fully extended, and there is no further entropic gain from expansion. These detailed molecular simulations are in agreement with SCFT models, which showed that favorable interactions between surfactant and polymer can lead to intercalated structures. ${ }^{6,10}$

Application of Shear. To determine the properties of the clay layers under processing conditions, we performed calculations to determine the minimum shear force required to separate clay layers. First, we consider the case where the surfactant and the surrounding polymer are chemically identical $\left(\mathrm{NC}_{1} 8_{2}\right.$ surfactant and PE polymer). For this model, we examine the clay tactoid at its lowest-free-energy clay sheet separation (28 Å) (see Figure 3a) and, for comparison, at a high separation of $44 \AA$ where the clay surface and surfactants of the two layers can be considered as noninteracting (Figure 3b).

In Figure 3c, we show the displacement as a result of different forces applied to the upper layer of our two-clay-layer system. At the lowest-energy separation, we find that the greater the force, the greater the shift of the upper platelet, and rupture, defined as the upper clay sheet moving more than 50 $\AA$, occurs at $0.0325 \mathrm{kcal} /(\mathrm{mol} \AA)$. When the clay sheets are noninteracting, in each case the applied force separates the clay sheets, and the distance traveled by the upper sheet in a specified time is proportional to the force applied (Figure 3d). These simulations indicate that there is significant entanglement of the surfactant molecules at the lowest-energy separation and a relatively large force is required to overcome these adhesive forces; resistance is completely removed when the sheets are far enough apart that the surfactant molecules do not interact. In Figure 3a, we highlight the considerable number of surfactant molecules that act as a bridge between the two clay surfaces at the lowest-energy clay separation. These bridges, and interactions between the surfactant molecules, resist the rupture of the clay tactoid; considerable force is required to desorb these molecules. 

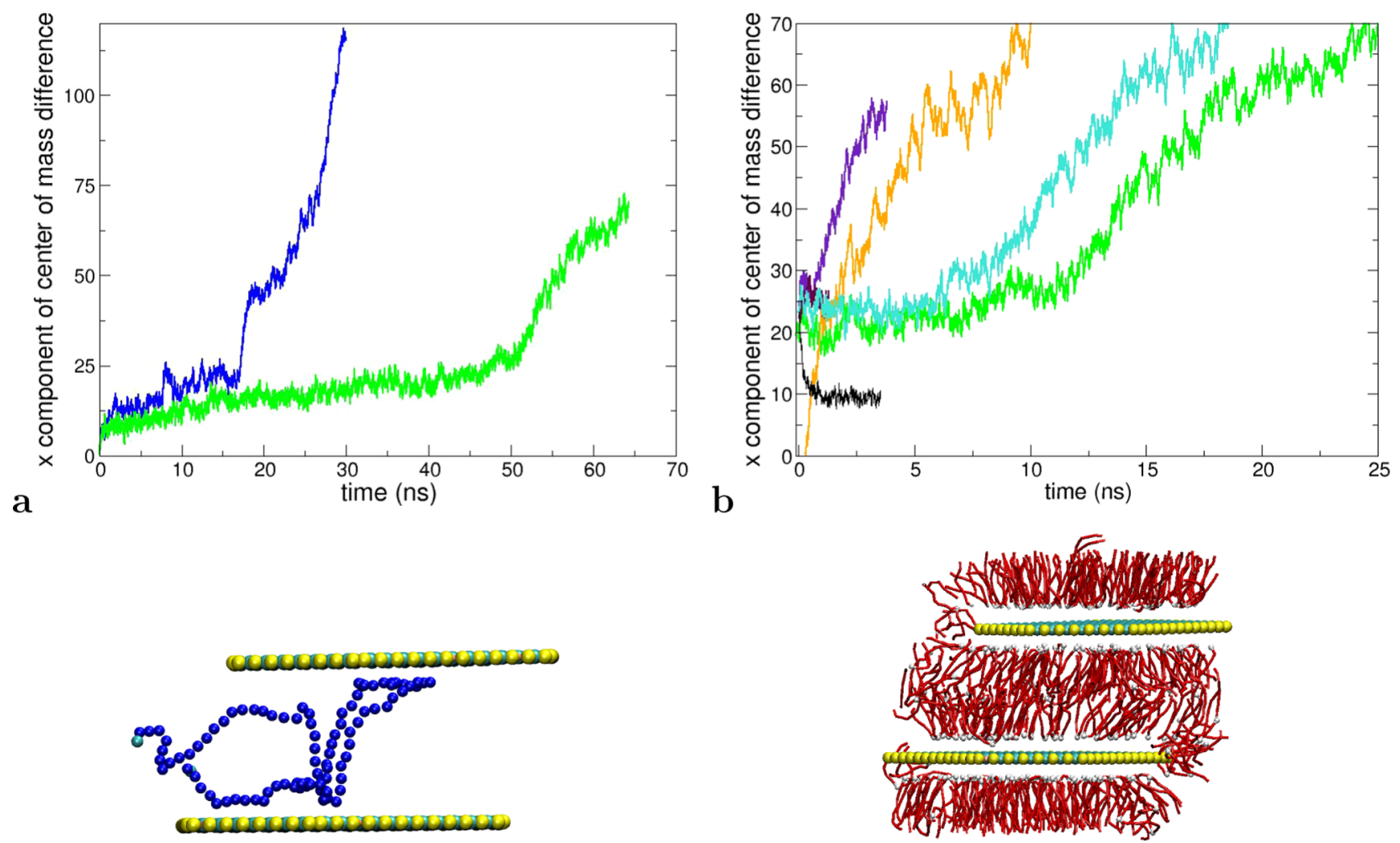

C

d

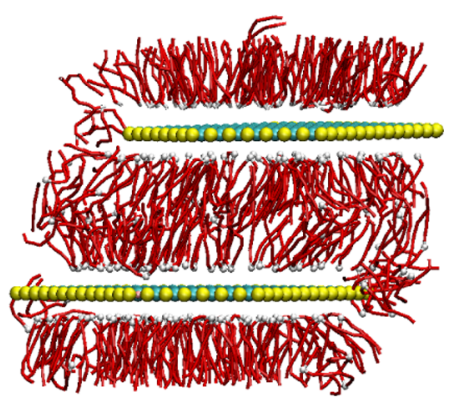

Figure 4. Shear-induced exfoliation of the $\mathrm{NC}_{2} 8_{2}$ surfactant and PEG polymer system. (a) Difference in $x$ component of the center of mass of clay layers at a $d$-spacing of $42 \AA$, a force added to each atom in the top clay layer of $0.02 \mathrm{kcal} /(\mathrm{mol} \AA$ ) (green line) and $0.03 \mathrm{kcal} /(\mathrm{mol} \AA$ ) (blue line). (b) Modified simulations, started from the unmodified simulation after $40 \mathrm{~ns}$ (the green curve in (a)). The colors are as follows: black, removal of bonded interactions in the polymer molecules; blue, removal of the bridging polymer molecules; brown, removal of bonded interactions between the surfactant molecules; orange, an unmodified simulation, starting at a greater clay-layer separation (55 A). (c) An example of a bridging polymer molecule, subsequently removed in the black line simulation in (b). (d) A snapshot from simulation where all bonded interactions (i.e., bonds, angles, and dihedrals) within the polymer molecules have been removed, thereby turning all polymer molecules into monomers (polymer molecules not shown in the visualization).

When polymer molecules intercalate into the clay gallery, the conformation of the polymer might also play a role in determining the resistance to shear exfoliation. These intercalated conformations are commonly described in terms of trains, loops, tails, and bridges: trains are in contact with a clay layer, loops dangle from a surface into solution between two trains, whereas tails are not in contact with the surface but extend into the melt. A bridge arises between two trains on different surfaces. A schematic diagram of these configurations is shown in Figure 1b. In the following section, we describe the influence of these different configurations on the resistance to shear exfoliation. In Figure 4a, we illustrate the displacement of the upper sheet under shear force for the surfactant-polymer combination of $\mathrm{N} 18_{2}$ surfactant and PEG polymer.

In contrast to the case where the surfactant backbone and the polymer molecules are chemically identical, with a hydrophilic polymer and hydrophobic surfactant, we find that the separation between the clay layers gradually increases as larger forces are applied to the upper clay layer. For the highest applied force $(0.03 \mathrm{kcal} /(\mathrm{mol} \AA))$, the separation increases in a stepwise fashion, until rupture occurs after $18 \mathrm{~ns}$. A similar trend is seen with an applied force of $0.02 \mathrm{kcal} /(\mathrm{mol} \AA)$, with shear-induced exfoliation occurring at $50 \mathrm{~ns}$. These forces are lower than the lowest force required to exfoliate the clay layer when the surfactant backbone and the polymer molecules are identical.

To analyze the factors inhibiting shear-induced exfoliation, we have modified the interactions present in our simulations to observe the effect on shear-induced exfoliation. We use as our starting structure the $0.02 \mathrm{kcal} /(\mathrm{mol} \AA)$ force simulation (the green line in Figure $4 \mathrm{a}$ ) at $40 \mathrm{~ns}$, which is approximately $10 \mathrm{~ns}$ before the separation in the clay layers increases dramatically due to the applied shear force. We removed polymer molecules, which form bridges across the interlayer (defined as polymer molecules with density within $9.5 \AA$ of both clay surfaces), an example of which is shown in Figure 4c. Restarting the simulation without the bridging polymers makes only a small difference to the shear-induced time profile, with separation still occurring $10 \mathrm{~ns}$ after the restart (blue line in Figure 4b). Thus, bridging polymer molecules only play a minor role in determining the resistance to shear exfoliation. Second, we removed all bonded interactions within polymer molecules, thereby turning all polymers into monomers units. This has the effect of removing all of the loops and bridges on each clay surface. In Figure $4 b$, we see that this process actually reduces the separation (black line); i.e., it increases the friction between the layers. It is, therefore, clear that the polymer molecules are not the main reason for the resistance to shear. To test the dependence on the surfactant interactions, we removed all 
bonds within the surfactant molecules, which led to a spontaneous increase in shear-induced separation (purple line in Figure $4 b$ ). Furthermore, we repeated the simulation using a snapshot from the free energy of separation simulation of the $\mathrm{N}_{1} 8_{2}$ surfactant and PEG polymer system with a $z$ separation of $55 \AA$, a distance at which there is no interaction between surfactants tethered to opposite surfaces, but there are bridging polymer molecules. In both cases, there is no resistance to shear-induced exfoliation, and the distance between the clay layers increases rapidly, again indicating that the interaction between the surfactant molecules is the determining factor in resisting shear-induced exfoliation. In Figure 4d, we illustrate the overlap between surfactant molecules tethered to opposite surfaces, thereby indicating their interaction, through a snapshot from one of our simulations.

\section{CONCLUSIONS}

We have examined the factors that affect the swelling of pretreated clay layers using multiscale methods to elucidate the processes that resist shear-induced exfoliation. Because they are initially added to promote expansion of clay layers, somewhat surprisingly we find the surfactant molecules dominate the mechanism by which clay layers resist exfoliation in their expanded state. For clays with surfactants that are chemically identical to the surrounding polymer, there is no thermodynamic driving force for polymer to intercalate, so the interlayer is dominated by strongly interacting surfactants. Only large shear forces are able to overcome these interactions. For clays with surfactants that possess favourable interactions with the surrounding polymer, although the thermodynamically stable $d$ spacing is much expanded through intercalation, the surfactant molecules from opposite surfaces interact strongly, in turn inhibiting shear-driven exfoliation. The force required to exfoliate is less than that for unintercalated clay platelets. Under shear, surfactant-surfactant interactions are gradually broken and the surfactants eventually untangle. By contrast, the polymer plays no role in resisting exfoliation.

We can compare the minimum shear force we have found in our simulations to those estimated to occur in processing conditions. According to the analysis of Borse and Kamal, ${ }^{23}$ the typical shear rates in extrusion processing are between 1 and $100 \mathrm{~s}^{-1}$, although higher rates can be found within an extruder. The viscosity of most polymers during extrusion is between 1000 and $2000 \mathrm{~Pa}$ s. Thus, the maximum available shear stress is of order $2 \times 10^{5} \mathrm{~N} / \mathrm{m}^{2}$. The surface area of our clay systems is approximately $140 \times 100 \AA^{2}$, and each clay layer contains 672 coarse-grained atoms. The minimum force required to break apart the clay tactoids in our simulations was $0.02 \mathrm{kcal} /(\mathrm{mol} \AA)$ per atom; this leads to a shear stress (i.e., total applied force/ surface area) of approximately $6.4 \times 10^{5} \mathrm{~N} / \mathrm{m}^{2}$, which is comparable to those found in extruders. Assuming that the force per atom required to exfoliate the clay layer is independent of the number of atoms in the clay layer, this value should be platelet size independent. Of course, our estimates are likely to be an upper bound, there may be other directions that require less force and, as we have shown, the duration of the applied force is important; lower forces may still cause shear-induced exfoliation over longer time scales than we can access here with the computing resources available to us.

In conclusion, we have shown that surfactants, while necessary to help initial intercalation, strongly resist the subsequent shear-induced exfoliation.

\section{ASSOCIATED CONTENT}

\section{Supporting Information}

The Supporting Information is available free of charge on the ACS Publications website at DOI: 10.1021/acsomega.8b00542.

Additional details regarding the simulation protocol used in this study and atomic density profiles perpendicular to the clay surface (PDF)

\section{AUTHOR INFORMATION}

\section{Corresponding Authors}

*E-mail: j.suter@ucl.ac.uk (J.L.S.).

*E-mail: p.v.coveney@ucl.ac.uk (P.V.C.).

ORCID

James L. Suter: 0000-0002-0149-7974

Peter V. Coveney: 0000-0002-8787-7256

\section{Notes}

The authors declare no competing financial interest.

\section{ACKNOWLEDGMENTS}

We acknowledge partial funding from the EU H2020 research innovation program for the ComPat project (www.compatproject.eu) under Grant agreement no. 671564. Simulations were run on ARCHER at Edinburgh Parallel Computing Centre (www.archer.ac.uk), on SuperMUC at Leibniz Supercomputing Centre (LRZ) (www.lrz.de), and on Prometheus at Cyfronet www.cyfronet.krakow.pl. Supercomputing time on ARCHER was provided by the U.K. Consortium on Mesoscale Engineering Sciences (UKCOMES) under U.K. EPSRC Grant no. EP/L00030X/1 and a Resource Allocation Panel award; access to SuperMUC and Prometheus came from supercomputing awards at LRZ and Cyfronet.

\section{REFERENCES}

(1) Suter, J. L.; Groen, D.; Coveney, P. V. Chemically Specific Multiscale Modeling of Clay-Polymer Nanocomposites Reveals Intercalation Dynamics, Tactoid Self-Assembly and Emergent Materials Properties. Adv. Mater. 2015, 27, 966-984.

(2) Suter, J. L.; Groen, D.; Coveney, P. V. Mechanism of Exfoliation and Prediction of Materials Properties of Clay-Polymer Nanocomposites from Multiscale Modeling. Nano Lett. 2015, 15, 81088113.

(3) Marzari, N. Materials modelling: The frontiers and the challenges. Nat. Mater. 2016, 15, 381-382.

(4) Chen, B.; Evans, J. R. G.; Greenwell, H. C.; Boulet, P.; Coveney, P. V.; Bowden, A.; Whiting, A. A critical appraisal of polymer-clay nanocomposites. Chem. Soc. Rev. 2008, 37, 568-594.

(5) Ray, S. S. Recent trends and future outlooks in the field of claycontaining polymer nanocomposites. Macromol. Chem. Phys. 2014, $215,1162-1179$.

(6) Ginzburg, V. V.; Weinhold, J. D.; Jog, P. K.; Srivastava, R. Thermodynamics of polymer-clay nanocomposites revisited: Compressible self-consistent field theory modeling of melt-intercalated organoclays. Macromolecules 2009, 42, 9089-9095.

(7) Paul, D. R.; Robeson, L. M. Polymer nanotechnology: Nanocomposites. Polymer 2008, 49, 3187-3204.

(8) Vaia, R. A.; Giannelis, E. P. Polymer Melt Intercalation in Organically-Modified Layered Silicates: Model Predictions and Experiment. Macromolecules 1997, 30, 8000-8009.

(9) Vaia, R. A.; Giannelis, E. P. Lattice model of polymer melt intercalation in organically-modified layered silicates. Macromolecules 1997, 30, 7990-7999.

(10) Balazs, A. C.; Singh, C.; Zhulina, E. Modeling the interactions between polymers and clay surfaces through self-consistent field theory. Macromolecules 1998, 31, 8370-8381. 
(11) Ginzburg, V. V.; Singh, C.; Balazs, A. C. Theoretical phase diagrams of polymer/clay composites: the role of grafted organic modifiers. Macromolecules 2000, 33, 1089-1099.

(12) Lyatskaya, Y.; Balazs, A. C. Modeling the Phase Behavior of Polymer- Clay Composites. Macromolecules 1998, 31, 6676-6680.

(13) Hackett, E.; Manias, E.; Giannelis, E. Molecular dynamics simulations of organically modified layered silicates. J. Chem. Phys. 1998, 108, 7410-7415.

(14) Hackett, E.; Manias, E.; Giannelis, E. Computer simulation studies of PEO/layer silicate nanocomposites. Chem. Mater. 2000, 12, 2161-2167.

(15) Lee, J. Y.; Baljon, A. R.; Sogah, D. Y.; Loring, R. F. Molecular dynamics study of the intercalation of diblock copolymers into layered silicates. J. Chem. Phys. 2000, 112, 9112-9119.

(16) Baljon, A. R.; Lee, J. Y.; Loring, R. F. Molecular view of polymer flow into a strongly attractive slit. J. Chem. Phys. 1999, 111, 90689072.

(17) Lee, J. Y.; Baljon, A. R.; Loring, R. F.; Panagiotopoulos, A. Z. Simulation of polymer melt intercalation in layered nanocomposites. J. Chem. Phys. 1998, 109, 10321-10330.

(18) Kreer, T. Polymer-brush lubrication: a review of recent theoretical advances. Soft Matter 2016, 12, 3479-3501.

(19) Binder, K.; Kreer, T.; Milchev, A. Polymer brushes under flow and in other out-of-equilibrium conditions. Soft Matter 2011, 7, 71597172 .

(20) Galuschko, A.; Spirin, L.; Kreer, T.; Johner, A.; Pastorino, C.; Wittmer, J.; Baschnagel, J. Frictional forces between strongly compressed, nonentangled polymer brushes: Molecular dynamics simulations and scaling theory. Langmuir 2010, 26, 6418-6429.

(21) Pastorino, C.; Binder, K.; Kreer, T.; Müller, M. Static and dynamic properties of the interface between a polymer brush and a melt of identical chains. J. Chem. Phys. 2006, 124, No. 064902.

(22) Bousmina, M. Study of Intercalation and Exfoliation Processes in Polymer Nanocomposites. Macromolecules 2006, 39, 4259-4263.

(23) Borse, N. K.; Kamal, M. R. Estimation of stresses required for exfoliation of clay particles in polymer nanocomposites. Polym. Eng. Sci. 2009, 49, 641-650.

(24) Bandyopadhyay, J.; Ray, S. S.; Scriba, M.; Wesley-Smith, J. A combined experimental and theoretical approach to establish the relationship between shear force and clay platelet delamination in melt-processed polypropylene nanocomposites. Polymer 2014, 55, 2233-2245.

(25) Heinz, H.; Vaia, R. A.; Farmer, B. L. Interaction energy and surface reconstruction between sheets of layered silicates. J. Chem. Phys. 2006, 124, No. 224713.

(26) Fu, Y. T.; Heinz, H. Cleavage energy of alkylammoniummodified montmorillonite and relation to exfoliation in nanocomposites: Influence of cation density, head group structure, and chain length. Chem. Mater. 2010, 22, 1595-1605.

(27) Liu, P. Polymer modified clay minerals: A review. Appl. Clay Sci. 2007, 38, 64-76.

(28) Losego, M. D.; Blitz, I. P.; Vaia, R. A.; Cahill, D. G.; Braun, P. V. Ultralow thermal conductivity in organoclay nanolaminates synthesized via simple self-assembly. Nano Lett. 2013, 13, 2215-2219.

(29) Rühle, V.; Junghans, C.; Lukyanov, A.; Kremer, K.; Andrienko, D. Versatile Object-Oriented Toolkit for Coarse-Graining Applications. J. Chem. Theory Comput. 2009, 5, 3211-3223.

(30) Müller-Plathe, F. Coarse-Graining in Polymer Simulation: From the Atomistic to the Mesoscopic Scale and Back. Chem. Phys. Chem. 2002, 3, 754-769.

(31) Roux, B. The calculation of the potential of mean force using computer simulations. Comput. Phys. Commun. 1995, 91, 275-282.

(32) Plimpton, S. Fast parallel algorithms for short-range molecular dynamics. J. Comput. Phys. 1995, 117, 1-19.

(33) Kumar, S.; Rosenberg, J. M.; Bouzida, D.; Swendsen, R. H.; Kollman, P. A. The weighted histogram analysis method for freeenergy calculations on biomolecules. I. The method. J. Comput. Chem. 1992, 13, 1011-1021. 